\title{
CUERPO EXTRAÑO SIMULANDO TUMOR RENAL
}

\author{
D. PASCUAL REGUEIRO, E. MALLÉN MATEO, M. ALLUÉ LÓPEZ, \\ Á. GARCÍA DE JALÓN MARTÍNEZ, Á. RONCALÉS BADAL, L.Á. RIOJA SANZ
}

Servicio de Urología. Hospital Universitario Miguel Servet. Zaragoza.

Actas Urol Esp. 28 (5): 390-392, 2004

\section{RESUMEN}

\section{CUERPO EXTRAÑO SIMULANDO TUMOR RENAL}

Un tumor renal de seis cm es encontrado en una ecografia prostática de control. Se indica una exéresis quirúrgica. Dicha tumoración presentó una mínima separación con el riñón. El riñón estaba únicamente comprimido por la masa la cual contenía una gasa quirúrgica en su interior de una cirugía previa hacía 35 años.

PALABRAS CLAVE: Cuerpo extraño. Tumor renal.

\section{ABSTRACT}

\section{FOREIGN BODY SIMULATING KIDNEY TUMOUR}

A six cm kidney tumour is found in a prostate control ultrasonography, in a 70 aged men. A chirurgical remove is indicated the tumour presented a very few separation of the kidney. The kidney was only compresed by the mass which contained a chirurgical gauze inside it from a previous surgery 35 years before.

KEY WORDS: Foreign body. Kidney tumour.

$\mathrm{U}$ na de las complicaciones presente en todo tipo de cirugías, ya sean urológicas, digestivas, ginecológicas etc., es el olvido de material quirúrgico, tanto desechable como inventariable dentro del campo quirúrgico ${ }^{8,12}$.

Guizás el material más frecuentemente implicado es el textil, en forma de gasas y compresas quirúgicas ${ }^{2,14}$, que tras ser utilizadas para secar, hacer compresión y hemostasia o simplemente para conseguir mejores exposiciones, desaparecen inadvertidamente en el campo quirúrgico.

Dichos materiales pueden permanecer silentes durante mucho tiempo o por el contrario manifestarse en forma de molestias abdominales persistentes de difícil filiación, s. febriles sin foco aparente etc. ${ }^{1,16}$.

Aunque la mortalidad por este motivo es muy baja, la morbilidad ${ }^{5,11}$ es altísima, implicando una reintervención quirúrgica para su extracción, muchas de las veces con el diagnóstico inicial de un proceso tumoral ${ }^{4,15}$.
A todo esto hay que añadir una serie de implicaciones ético-morales y jurídicas no despreciables, que nos obligan a ser extremadamente meticulosos al respecto ${ }^{13}$, llevando a cabo un correcto contaje del material, sobre todo del textil, antes de proceder al cierre.

\section{CASO CLÍnICO}

Se trata de un paciente de 70 años, el cual refiere sintomatología de tracto urinario inferior en forma de inicio de aumento de frecuencia miccional nocturna, ligera disuria, así como leve disminución del calibre miccional.

Ante esta sintomatología prostática incipiente, es remitido a su urólogo de zona, el cual realiza un tacto rectal (adenoma II), y solicita una determinación de PSA y una ecografía abdominal para la siguiente consulta.

El paciente mostraba como únicos antecedentes una intervención sobre el ojo izquierdo como consecuencia de un traumatismo, y en el plano 
urológico una ureterolitectomía del tercio superior del uréter hacía 35 años en otro centro, a través de una lumbotomía.

En la siguiente consulta, y encontrándose el paciente totalmente asintomático, se determina en el estudio ecográfico la existencia de una masa parahiliar en el riñón izquierdo de unos $6 \mathrm{~cm}$ de diámetro, un riñón derecho normal, una próstata de 35 cc, así como un PSA dentro de los rangos normales.

Ante estos hallazgos y la sospecha de una tumoración renal, se realiza un TAC con el resultado de imagen parapiélica izquierda, de 5-6 cm de diámetro, de aspecto sólido, que se extiende desde el hilio renal hasta por debajo del polo inferior, con compresión del riñón y con un dudoso plano graso de separación con el mismo, calcificaciones en su interior y muy baja vascularización (Fig. 1).

Con el diagnóstico de masa renal se realiza un estudio de extensión que resulta negativo, planteándose la revisión quirúrgica y exéresis de la masa y/o nefrectomía según hallazgos.

Quince días después se lleva a cabo una lumbotomía izquierda.

Una vez iniciadas las maniobras de disección renal, se identifica claramente la masa comprimiendo riñón, pero con claro plano de disección entre ambos. Dada las dudas de la naturaleza de
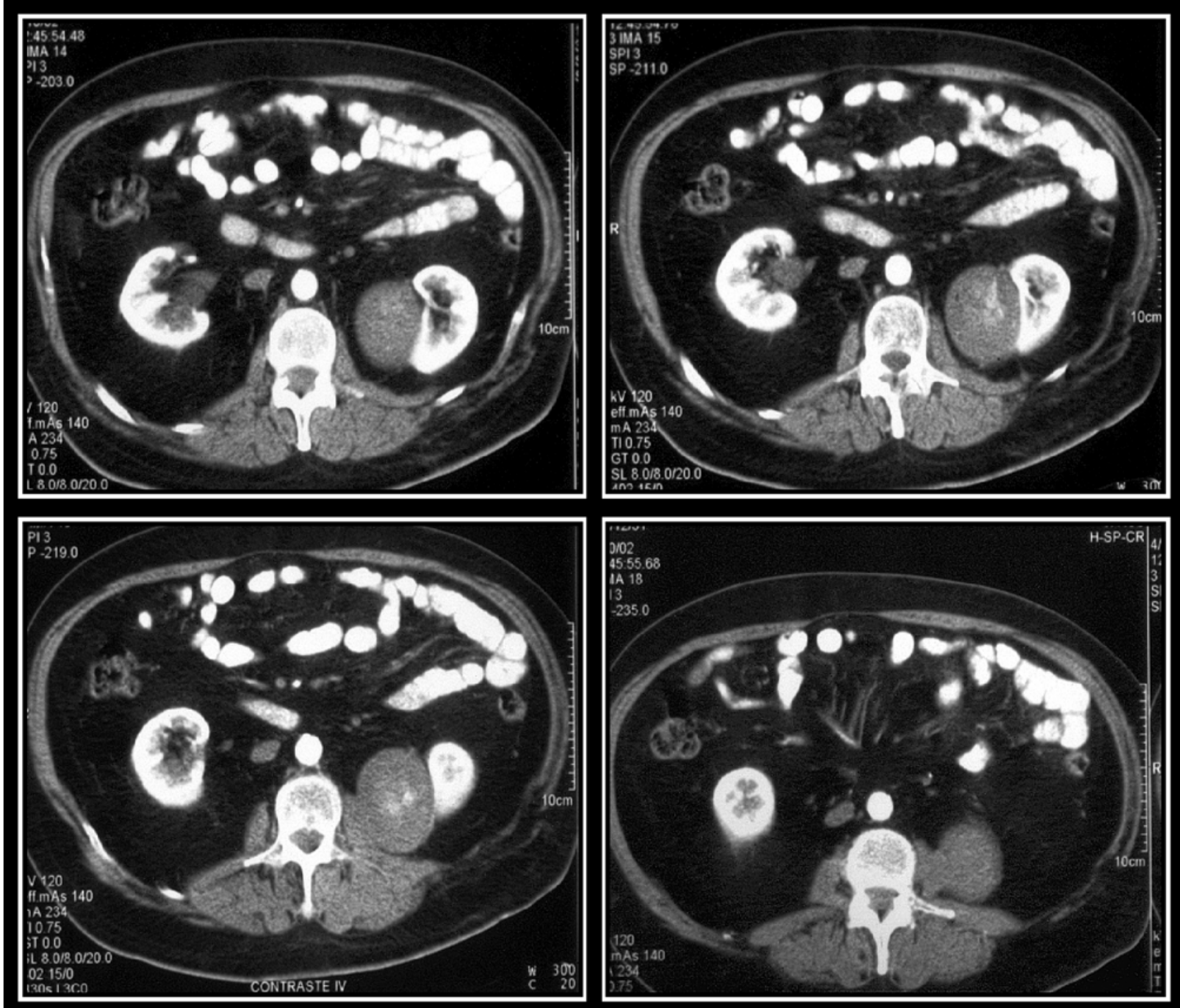

FIGURA 1. Imagen del TAC en la que se aprecia la tumoración parahiliar izquierda de caracteristicas sólidas y calcificaciones en su interior, con un tamaño de 5-6 cm de diámetro máximo. 
la masa, se realiza una biopsia intraoperatoria obteniéndose fibras textiles.

Ante la evidencia de que se estaba ante una gasa de la intervención previa, se realiza la exéresis de la totalidad de la tumoración conservando la unidad renal.

Una vez realizada la exéresis de la masa, ésta es abierta encontrándose en su interior fragmentos deshilachados de una o varias gasas, con material de aspecto caseoso a su alrededor (Fig. 2).

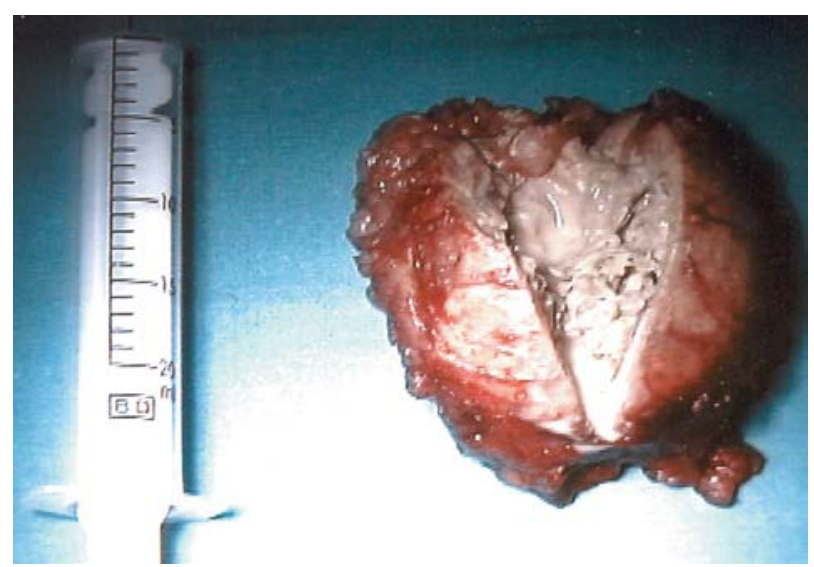

FIGURA 2. Imagen macroscópica de la pieza quirúrgica abierta en sentido longitudinal, mostrando un interior con material de aspecto caseoso y fibras textiles.

El análisis anatomopatológico de la pared de la tumoración hablaba de material hialino con tendencia a la colagenización, y reacción inflamatoria sobre todo a expensas de histiocitos.

\section{COMENTARIO}

Como vemos, nos encontramos ante un paciente que hace 35 años, cuando no existía el marcaje radiológico de las gasas y compresas quirúrgicas, sufrió una cirugía renal en la que inadvertidamente se quedaron olvidadas en el campo quirúrgico una o varias gasas.

Después de todo este tiempo asintomático, casualmente se realiza un estudio ecográfico que pone de manifiesto la presencia de una masa renal.

Se trata pues de una larga evolución ${ }^{6,9,10}$ en ausencia de síntomas, en la que quizás si no hubiéramos realizado la ecografía, el cuerpo extraño hubiera pasado desapercibido y quizás hubiéramos evitado la morbilidad de un acto quirúrgico.
Esto, por supuesto, no exime de la responsabilidad de evitar con todos los medios posibles, sobre todo con un contaje exhaustivo de gasas y compresas quirúrgicas, la repetición de estas situaciones, dado que en gran parte de las mismas existe una clínica, por otra parte insidiosa y mantenida, $\mathrm{y}$ posibles y graves complicaciones asociadas.

\section{REFERENCIAS}

1. SCHELHAAS E, MASTBOOM WJ.: A retained gauze as a cause of unexplained abdominal complaints. Ned Tïdschr Geneeskd 2002 feb 9; 146 (6): 241-245. Comentado en: Ned Tijdschr Geneeskd 2002 apr 27; 146 (17): 825; discussion 825. Ned Tijdschr Geneeskd 2002 feb 9; 146 (6): 245-246.

2. BALLESTEROS SAMPOL JJ, ALAMEDA QUITLLET F, PARES PUNTAS ME.: Tres raros casos de textiloma tras cirugia renal. Revisión de la literatura. Arch Esp Urol 2002 jan-feb; 55 (1): 25-29.

3. DÍAZ PLASENCIA J, TANTALEAN RAMELLA E, BALMACEDA FRASELLE T, HONORIO HORNA CE, BARANDIARAN DEJO MA, VILELA GUILLEN E, QUIROZ EY.: Foreign body in abdomen: report of a case. Rev Gastroenterol Peru 2001 jul-sep; 21 (3): 229233.

4. FUKATSU T, TAJIMA K, SAITOU K.: Two cases of abdominal masses caused by foreign bodies which were preoperatively diagnosed as urachal abscess. Hinyokika Kiyo 2000 may; 46 (5): 341-344.

5. AWE JA.: Intestinal obstruction from a forgotten artery forceps: a case report. West Afr J Med 2000 jul-sep; 19 (3): 235-237.

6. SKYBA VV, TARAPON OIU, IATSYSHYN IV, IAVORS'KYI II.: Longterm stay of foreign body in abdominal cavity. Klin Khir 2000 nov; (11): 62 .

7. GOKALP A, MARALCAN G.: Acta forgotten rubber drain in the abdomen (case report). Chir Hung 1999; 38 (3-4): 343-347.

8. GONZÁLEZ OJEDA A, RODRÍGUEZ ALCÁNTARA DA, ARENAS MÁRQUEZ H, SÁNCHEZ PÉREZ-VERDIA E, CHAVEZ PÉREZ R, ÁLVAREZ QUINTERO R, PEREA SÁNCHEZ A.: Retained foreign bodies following intra-abdominal surgery. Hepatogastroenterology 1999 mar-apr; 46 (26): 808-812.

9. QUANTZ MA, BROWN R. Late presentation of an intra-abdominal foreign body. Can J Surg 1997 aug; 40 (4): 305-307. Comentado en: Can J Surg 1997 aug; 40 (4): 249-250.

10. EMERICH J, KONEFKA T, DUDZIAK M, MADRZAK J.: A case of a foreign body-surgical linen left in the abdominal cavity 25 years. Earlier Ginekol Pol 1996 may; 67 (5): 274-276.

11. CHEN B, HALL JB, BRUNER JP.: Intraperitoneal foreign body as a cause of acute abdomen in pregnancy. Am J Perinatol 1995 mar; 12 (2): 100-101.

12. GATAULLIN NG, VLASOV AF, CHUKHRIENKO DP, SERNIAK PS, ZAVGORODNII LG.: Postoperative foreign bodies in the abdominal cavity. Vestn Khir Im I I Grek 1977 sep; 119 (9): 118-123.

13. SUBBOTIN VM, DAVIDOV MI.: The reasons for leaving foreign bodies in the abdominal cavity and the prevention of this complication. Vestn Khir Im I I Grek 1998; 157 (4): 79-84.

14. SLIM K, BEN SLIMANE T, DZIRI C, MZABI R. Textile foreign bodies retained in the abdomen. Ann Chir 1989; 43 (4): 285-288.

15. NEKAEV OK, PEREKUPENKO AG.: Foreign body in the abdomen, diagnosed as cancer of the mesentery. Klin Khir 1979 feb; (2): 74.

16. KELTNER RM Jr.: Late clinical manifestations of foreign bodies left in the abdomen. Case reports. Mo Med 1967 jun; 64 (6): 495496 passim.

Dr. D. Pascual Regueiro

Avda. Compromiso de Caspe, 27-29, 2ํㅡ A

50002 Zaragoza

(Trabajo recibido el 4 marzo de 2003) 